\title{
Usage of multi-objective genetic and multi-objective differential evolution algorithms on energy and spectral efficiencies in massive MIMO systems
}

\author{
Burak Kürşat Gül * and Necmi Taşpınar \\ Department of Electrical and Electronics Engineering, Erciyes University, Kayseri, Turkey.
}

Publication history: Received on 22 September 2020; revised on 28 September 2020; accepted on 29 September 2020

Article DOI: https://doi.org/10.30574/gjeta.2020.5.1.0079

\begin{abstract}
There is a significant increase in the use of wireless communication and it is expected that this increase will continue progressively. In the near future, cellular network technologies are expected to be capable of increasing the area throughput hundreds of times in order to cope with the increase in data traffic. Increasing spectral efficiency (SE) with massive multi-input multi-output (Massive MIMO) systems is one of the main methods used to meet these expectations. SE means the amount of information transmitted successfully with each complex sample. Increasing the transmission power and the number of active antennas while increasing the SE increases the amount of energy consumed to very high levels. The fact that high energy consumption is harmful to the environment and costly makes it important to increase energy efficiency (EE). Various studies are carried out with the aim of bringing optimum levels of the SE and EE parameters which has trade-off between each other. Multi-objective intelligent optimization techniques are applied on the trade-off for detecting optimum SE-EE values. In this paper, multi-objective genetic algorithm (MOGA) and multiobjective differential evolution algorithm (MODEA) are used to obtain optimum values of certain factors (amount of transmit power, number of active antennas and number of user equipments). At the last stage, the calculations made for all values of the mentioned factors and the optimization results (performed in a relatively short time compared to these calculations) are shown on the same graph.
\end{abstract}

Keywords: Massive MIMO; Spectral efficiency; Energy efficiency; Multi-objective genetic algorithm; Multi-objective differential evolution algorithm

\section{Introduction}

Wireless communication technologies are becoming widespread thanks to the facilities and possibilities they provide. Today, there has been a demand for wireless communication almost anytime and anywhere and it is predicted that these demands will increase even more. This expected increase may cause the wireless data transmission traffic to increase very rapidly. In order to cope with the increase in data traffic, cellular network technologies will need to have the capacity to increase area throughput (TR) hundreds of times [1].

One of the most practical ways to increase area throughput is to increase spectral efficiency (SE). Massive multipleinput multiple-output (Massive MIMO) system is one of the preferred methods in studies to increase spectral efficiency $[2,3,4]$. This system is based on the principle of using hundreds or thousands of antennas per base station (BS), unlike conventional MIMO methods [5].

The spectral efficiency of an encoding or decoding algorithm is equivalent to the average number of bits for each complex-valued sample of information [bit/s/Hz] [1]. Spectral efficiency can be increased by some operations such as increasing the transmission power, increasing the number of receiving antennas at the base station or using the space division multiple access (SDMA) method during transmission.

\footnotetext{
${ }^{*}$ Corresponding author: Burak Kürşat Gül

Department of Electrical and Electronics Engineering, Erciyes University, Kayseri, Turkey.

Copyright $(2020$ Author(s) retain the copyright of this article. This article is published under the terms of the Creative Commons Attribution Liscense 4.0.
} 
Increasing the transmission power and the number of active antennas in an uncontrolled way can increase the amount of energy consumed to very high levels. This situation is considered to be an important problem because it is both harmful to the environment and high cost [6]. While increasing SE, less energy consumption can be achieved by increasing energy efficiency (EE). Energy efficiency refers to the number of bits transmitted successfully using unit energy [bit/Joule]. In studies examining SE and EE together, it is observed that SE increases continuously as the transmission power and the number of active antennas increase, while EE increases up to a point and then decreases. So, there is a trade-off between SE and EE.

Figure 1 shows an example where all ideal results obtained by using different numbers of antennas and different transmission power are collected in a single graphic. The curve indicated in red on the figure represents the true Pareto curve. This curve is created by the combination of all elements that cannot be dominated by other elements. Since it is non-convex non-concave and the problem contains continuous variables, the calculation of the Pareto curve involves a lot of processing load. Therefore, it may be preferred to find the values as close to this curve as possible in a much shorter time by using optimization methods instead of calculating this curve.

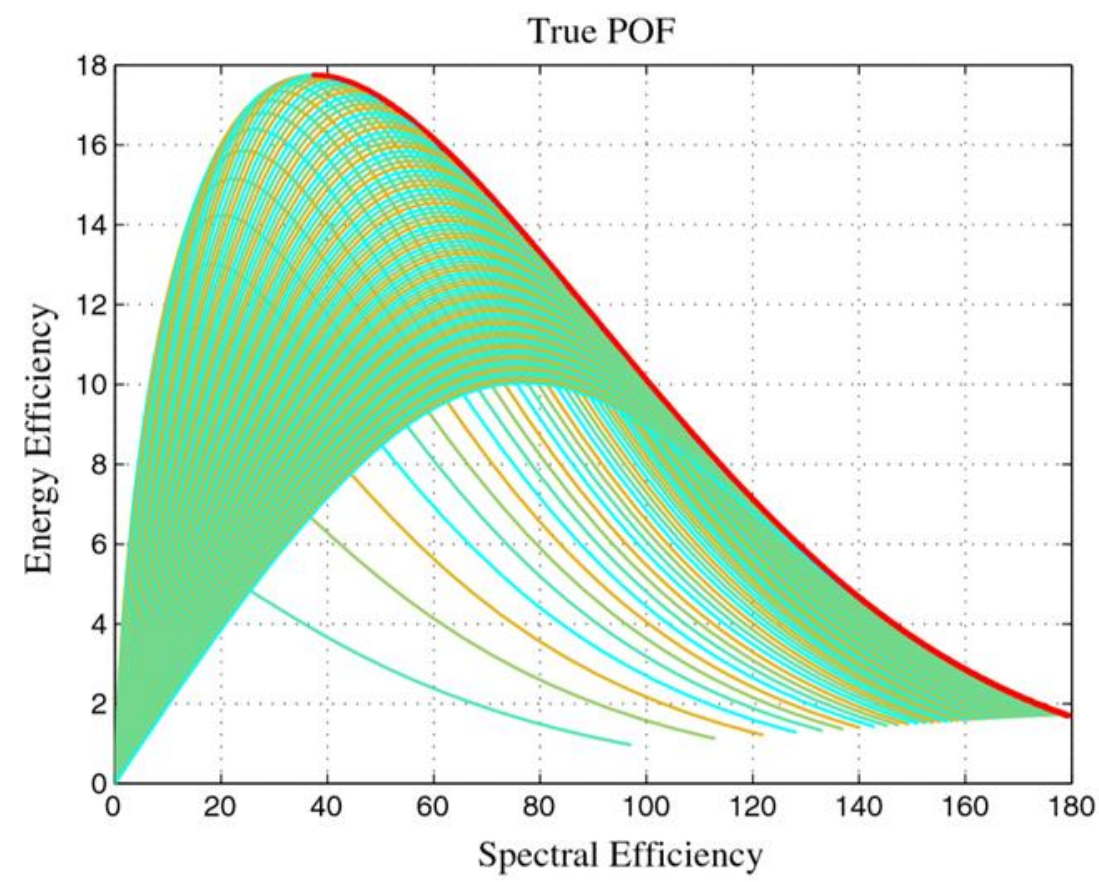

Figure $1 \mathrm{SE}-\mathrm{EE}$ values in cases where various antenna numbers and transmission power are used [7]

Intelligent optimization techniques are one of the optimization techniques whose usage area is constantly increasing. They have remarkable features such as simple and flexible structures, not requiring complex mathematical expressions, high accuracy and fast results, and finding local optimum. There are successful studies in which intelligent optimization techniques are used in SE-EE trade-off [7, 8]. Finding the optimum values of the SE-EE trade-off is classified as multiobjective optimization (MO0) problems [8].

In this paper, samples of the most appropriate combinations of certain factors were found by using multi-objective genetic algorithm (MOGA) and multi-objective differential evolution algorithm (MODEA). The samples obtained were shown on the same graph with all the calculated results and the results were examined.

\section{Material and methods}

Since the main goal of the study is to determine the situations where SE and EE are high at the same time, the problem is defined as MOO. In the first place, the results are calculated and stored for all the values that can be taken at certain range by the 3 effective factors (amount of transmit power, number of active antennas and number of user equipments) in SE and EE calculations. Then, optimum values are obtained on the SE - EE trade-off and non-dominated ones are determined. At this stage, multi-objective genetic algorithm and multi-objective differential evolution algorithm are used. 


\subsection{Creating Telecommunication Environment}

The parameters used in the cellular network example creation stage and the values they take are given in Table 1 . The structures used in the examples are shown in Figure 2.

Table 1 System parameters of the examples

\begin{tabular}{|c|c|}
\hline Parameter & Value \\
\hline Network layout & Square pattern (wrap-around) \\
\hline Number of cells & $0.25 \mathrm{~km} \mathrm{x} 0.25 \mathrm{~km}$ \\
\hline Cell area & $M$ \\
\hline Number of antennas per BS & $K$ \\
\hline Number of user equipments (UEs) per cell & $\Upsilon=-148.1 \mathrm{~dB}$ \\
\hline Channel gain at 1 km & $\alpha=3.76$ \\
\hline Pathloss exponent & $\sigma_{\mathrm{sf}}=10$ \\
\hline Shadow fading (standard deviation) & $B=20 \mathrm{MHz}$ \\
\hline Bandwidth & $-94 \mathrm{dBm}$ \\
\hline Receiver noise power & $20 \mathrm{dBm}$ \\
\hline Uplink transmit power & $20 \mathrm{dBm}$ \\
\hline Downlink transmit power & $\tau_{c}=200$ \\
\hline Samples per coherence block & $f=1,2 \mathrm{or} 4$ \\
\hline Pilot reuse factor & $\tau_{p}=f K$ \\
\hline Number of uplink pilot sequences & \\
\hline
\end{tabular}

Each cell is placed to cover an area of $0.25 \mathrm{~km}$ x $0.25 \mathrm{~km}$ as shown in Figure 2. Users will be randomly distributed at a distance of at least 35 meters from the base station and other users.

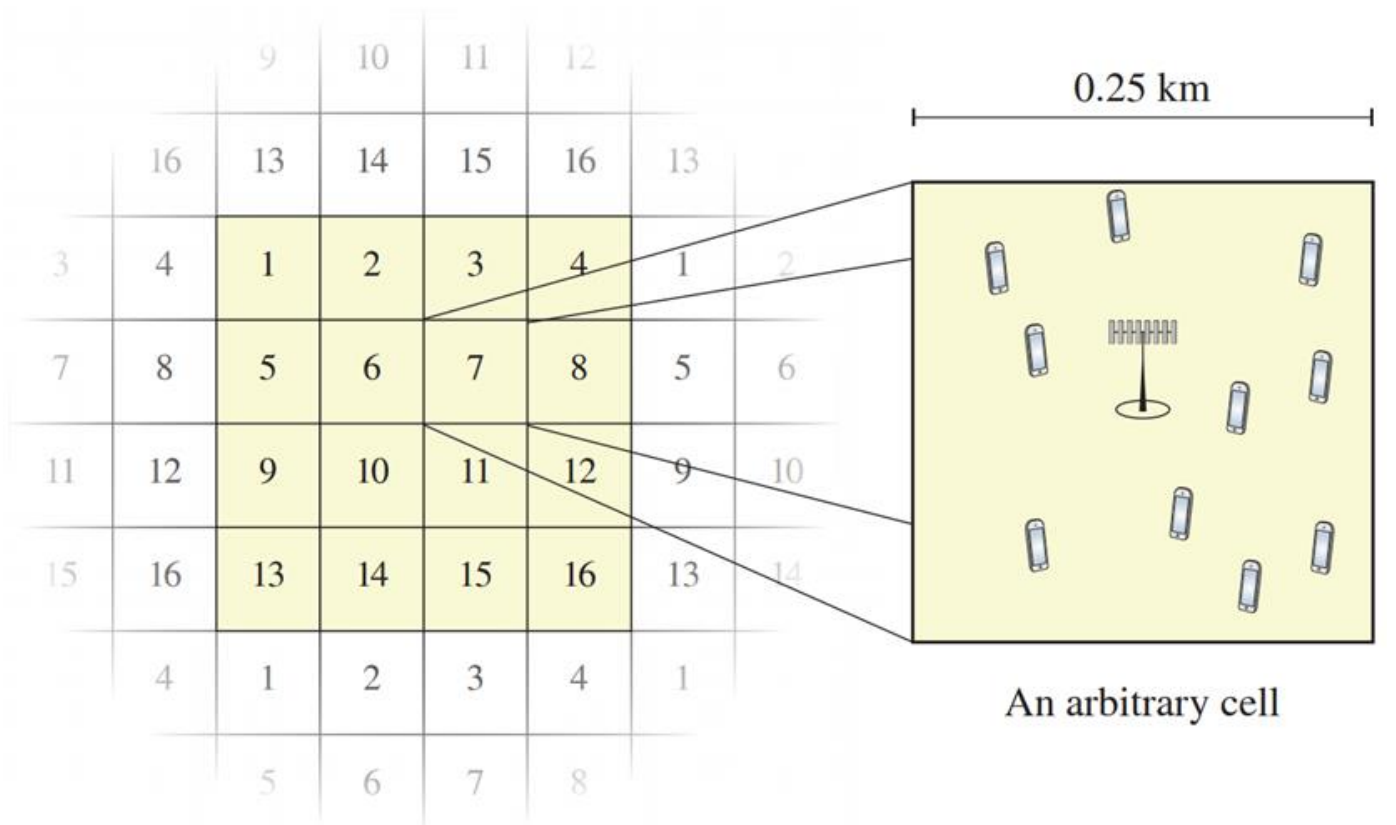

Figure 2 Illustration of cell locations and an arbitrary cell [1]

After the sample cells are created, spatial correlation matrices and the average channel gain matrix of all channels are created. Afterwards, channel realization and channel estimation of all these channels are made. All channels are considered as correlated Rayleigh fading and minimum mean-squared error (MMSE) estimator technique is used. 


\subsection{Spectral Efficiency}

The calculation of the spectral efficiency of an encoding or decoding algorithm is based on finding the spectral efficiencies of the uplink (UL) and downlink (DL) transmissions separately and adding these two values in certain ratios. In this study, the UL ratio was determined as $1 / 3$ and the DL ratio as $2 / 3$. The UL spectral efficiency is based on each base station detecting the information signal by linear receive combination. Calculating DL capacity is more difficult than calculating UL capacity because it has multiple limits and none of them is permanently selectable. Therefore, these two limits are calculated for each situation and the larger one is preferred.

\subsection{Energy Efficiency}

After SE calculations, calculations of EE values are performed depending on SE. Power parameters to be used in EE calculations and their values are given in Table 2.

Table 2 Power parameters of the examples

\begin{tabular}{|c|c|}
\hline Parameter & Value \\
\hline Fixed power: $P_{\mathrm{FIX}}$ & $10 \mathrm{~W}$ \\
\hline Power for BS local oscillator: $P_{\mathrm{LO}}$ & $0.2 \mathrm{~W}$ \\
\hline Power per BS antennas: $P_{\mathrm{BS}}$ & $0.4 \mathrm{~W}$ \\
\hline Power per UE: $P_{\mathrm{UE}}$ & $0.2 \mathrm{~W}$ \\
\hline Power for data encoding: $P_{\mathrm{COD}}$ & $0.1 \mathrm{~W} /(\mathrm{Gbit} / \mathrm{s})$ \\
\hline Power for data decoding: $P_{\mathrm{DEC}}$ & $0.8 \mathrm{~W} /(\mathrm{Gbit} / \mathrm{s})$ \\
\hline BS computational efficiency: $L_{\mathrm{BS}}$ & $75 \mathrm{Gflops} / \mathrm{W}$ \\
\hline Power for backhaul traffic: $P_{\mathrm{BT}}$ & $0.25 \mathrm{~W} /(\mathrm{Gbit} / \mathrm{s})$ \\
\hline
\end{tabular}

Energy efficiency is calculated by dividing the area throughput by the sum of effective transmit power (ETP) and circuit power (CP).

\subsection{Intelligent Optimization Algorithms}

In this paper, multi-objective versions of genetic algorithm and differential evolution algorithm are preferred. These algorithms have been modified to calculate two cost values (SE and EE) at the same time.

\subsubsection{Multi-objective Genetic Algorithm}

Similar to the genetic algorithm, the main stages are crossover and mutation. After the crossover stage, where the properties of two of the existing elements are blended, small changes are applied to the result in the mutation stage. After these two basic operations, the detected non-dominated elements are replaced with the old elements randomly selected. In this study, default probability of crossover, default probability of mutation and default mutation rate were determined as $70 \%, 40 \%$ and $2 \%$, respectively.

\subsubsection{Multi-objective Differential Evolution Algorithm}

In this algorithm, all existing elements are updated sequentially in each iteration. The updated element is compared with a temporary element created by blending the properties of three other elements. The blending stage is carried out by multiplying the differences of the values of the determined second and third elements by random coefficients and then adding them with the values of the first element. SE and EE calculations are made for the new element obtained and it is checked whether it can dominate the old element under consideration. If it can dominate the old element, it replaces it. These processes are continued until the termination condition is met. The default crossover percentage is 20 for this algorithm.

\section{Results and discussion}

The simulations performed with the default crossover ratios and probabilities are given in Figure 3, and an example in which the crossover amount is increased is given in Figure 4. While the calculation results of all possible values are shown with green curves, non-dominated values determined by intelligent optimization algorithms are shown with magenta asterisks. 


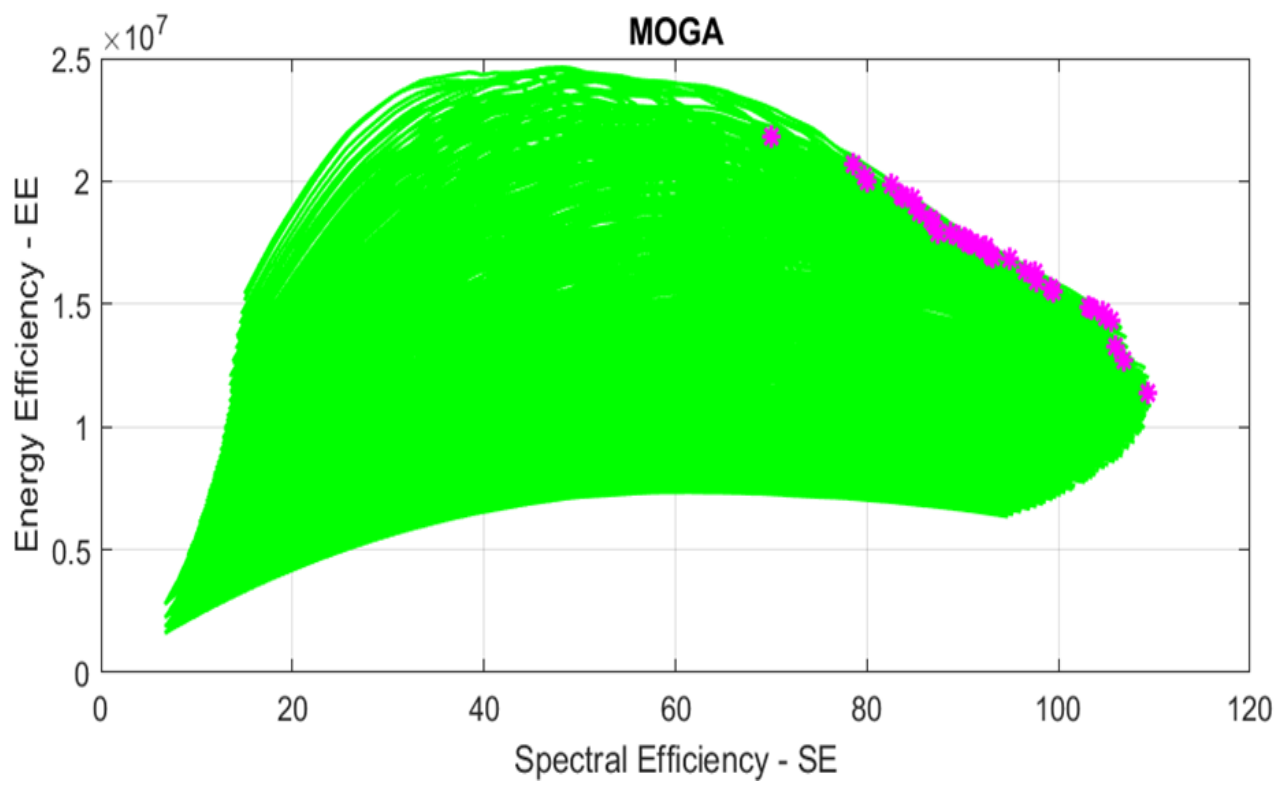

Figure 3a First sample of MOGA for SE-EE trade-off

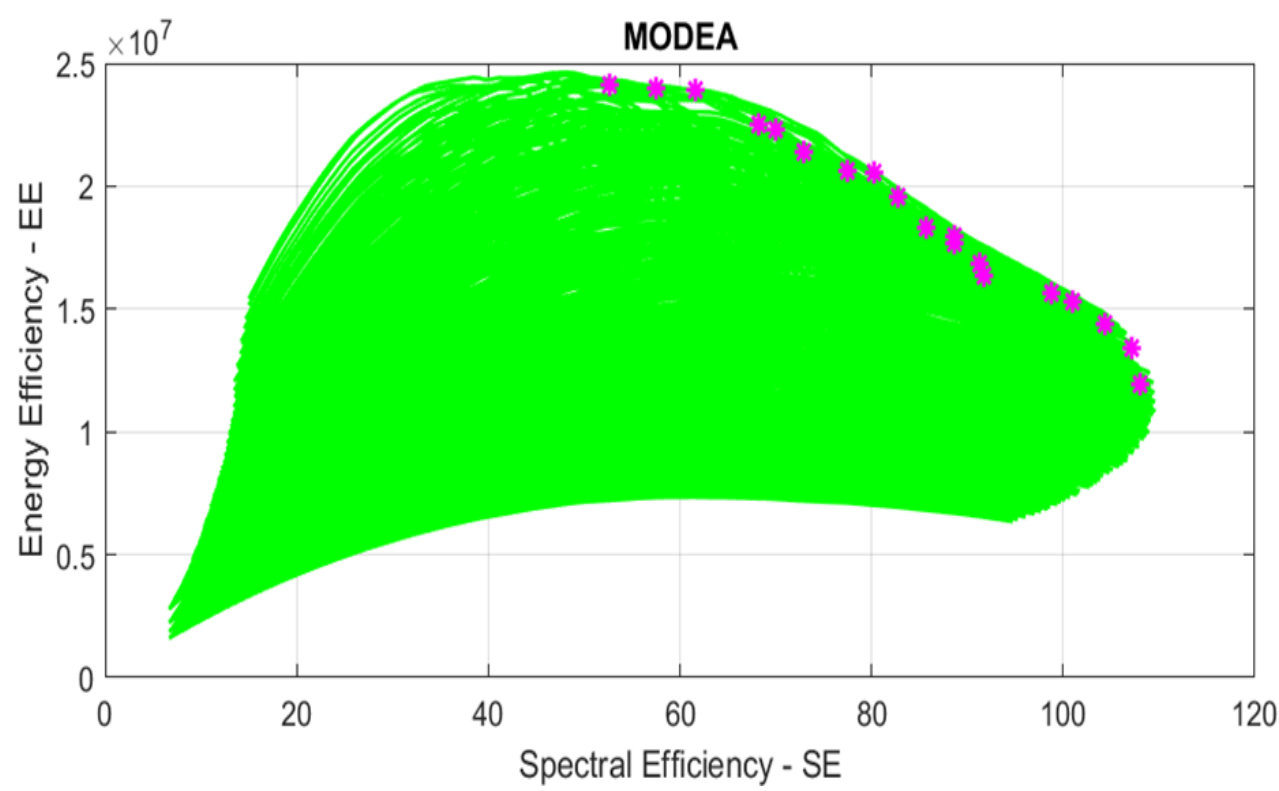

Figure 3b First sample of MODEA for SE-EE trade-off

When the solutions obtained with MOGA and MODEA are compared, it is seen that MODEA is better distributed on the true Pareto curve, for example, solutions which EE is maximum could not be found with MOGA. On the other hand, MOGA can detect more number and higher-value results.

The example of MOGA which the crossover rate is increased from 2 percent to 8 percent is shown in Figure 4a, and the example of MODEA which the crossover probability is increased from 20 percent to 40 percent is shown in Figure $4 \mathrm{~b}$. 


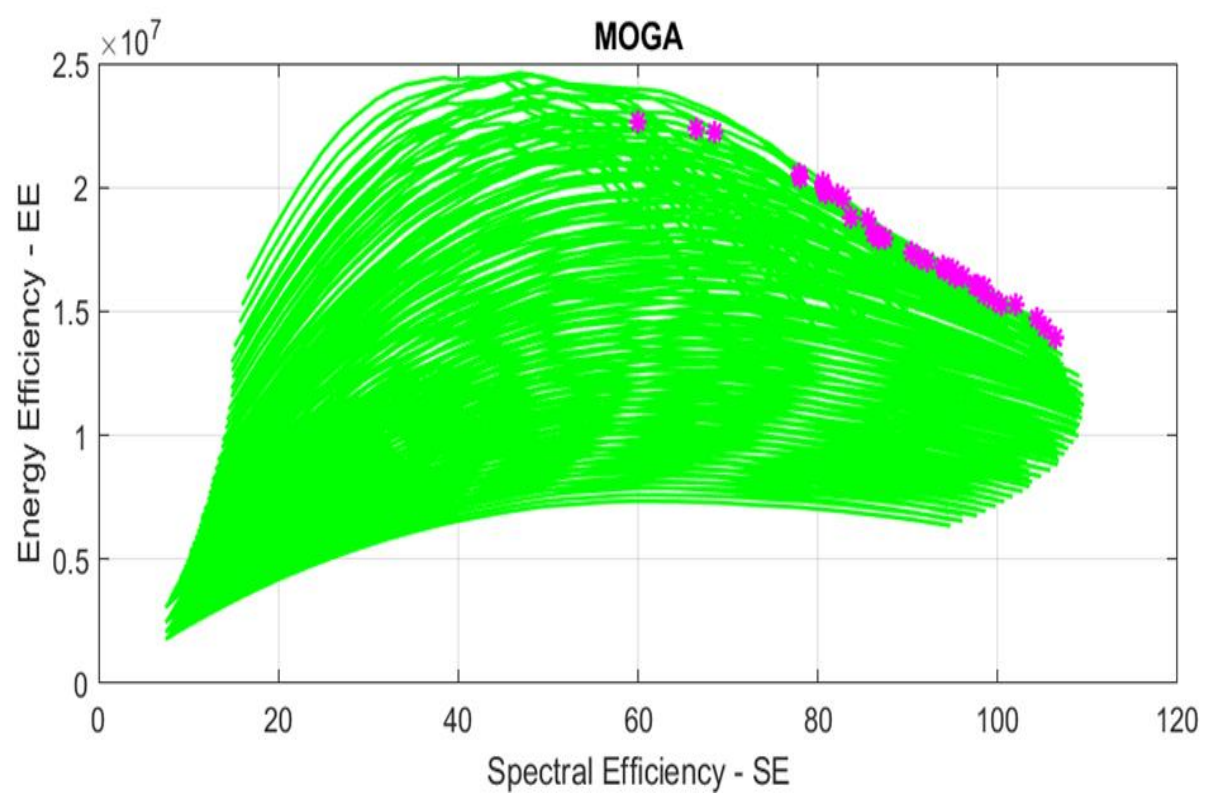

Figure 4a Second sample of MOGA for SE-EE trade-off

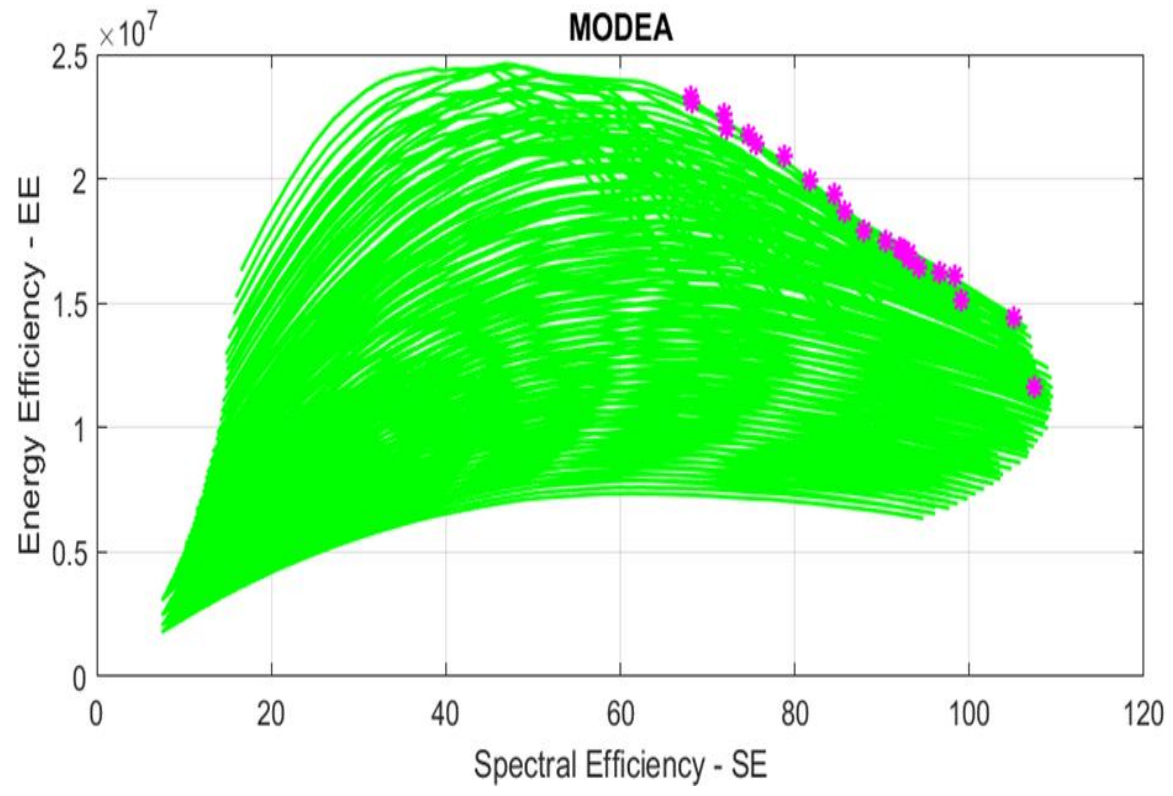

Figure 4b Second sample of MODEA for SE-EE trade-off

Although the increase in the rate or probability of crossover creates a positive effect on both algorithms, the performance increase is much higher in MODEA. It is seen that, the distributions on the curve increases in MOGA also MODEA can detect higher-value results by these changes.

\section{Conclusion}

It is seen that values which at acceptable proximity to the true Pareto curve are determined by using multi-objective genetic algorithm and multi-objective differential evolution algorithm. These processes, which are completed in a very short time compared to determining the optimum solutions among all the calculated results, can be used in the aforementioned problem. Based on the examples given to the effect of crossover parameters, it is concluded that by changing the other parameters of the algorithms, the results can be further improved. 


\section{Compliance with ethical standards}

\section{Acknowledgments}

The research is self-sponsored and did not receive grant from any organization.

\section{Disclosure of conflict of interest}

There is no conflict of interest declared on this research article.

\section{References}

[1] Björnson E, Hoydis J, Sanguinetti L. Massive MIMO Networks: Spectral, Energy and Hardware Efficiency. Foundation and Trends in Signal Processing. 2017; 11(3-4): 154-655.

[2] He Q, Xiao L, Zhong X, Zhou S. Increasing the Sum-throughput of Cells with a Sectorization Method for Massive MIMO. IEEE Communications Letters. 2014; 18(10): 1827-1830.

[3] Zhang B, Tian Y, Wang W. On the Downlink Throughput Capacity of Hybrid Wireless Networks with Massive MIMO. Eurasip Journal on Wireless Communications and Networking. 2018; (1) 110: 26086-26091.

[4] Yang G, Ho CK, Zhang R, Guan YL. Throughput Optimization for Massive MIMO Systems Powered by Wireless Energy Transfer. IEEE Journal on Selected Areas in Communications. 2015; 33(8), 1640-1650.

[5] Björnson E, Larsson EG, Debbah M. Massive MIMO for Maximal Spectral Efficiency: How Many Users and Pilots Sholud Be Allocated? IEEE Transactions on Wireless Communications. 2015; 15(2): 1293-1308.

[6] Fehske A, Fettweis G, Malmodin J, Biczok G. The Global Footprint of Mobile Communications: The Ecological and Economic Perspective. IEEE Transactions on Wireless Communications. 2011; 49(8): 55-62.

[7] Hei Y, Zhang C, Song W, Kou Y. Energy and Spectral Efficiency Tradeoff in Massive MIMO Systems with Multiobjective Adaptive Genetic Algorithm. Springer-Verlag GmbH Germany. 2018.

[8] Liu Z, Du W, Sun D. Energy and Spectral Efficiency Tradeoff for Massive MIMO Systems with Transmit Antenna Selection. IEEE Transactions on Vehicular Technology. 2017; 66(5): 4453-4457. 